\title{
A VIVÊNCIA DA FAMILIA NO CUIDADO DOMICILIAR À CRIANÇA COM NECESSIDADES ESPECIAIS DE SAÚDE
}

\author{
FAMILY EXPERIENCE IN HOUSEHOLD CARE FOR CHILDREN \\ WITH SPECIAL NEEDS OF HEALTH
}

\section{LA VIVENCIA DE LA FAMILIA EN EL CUIDADO DOMICILIAR AL NIÑO CON NECESIDADES ESPECIALES DE SALUD}

\author{
Kamilla Milione Nogueira Reis* \\ Gabriela Vilaça Alves ${ }^{* *}$ \\ Thaís Araújo Barbosa ${ }^{* * *}$ \\ Gabriela de Oliveira LOMBA ${ }^{* * * *}$ \\ Patrícia Pinto Braga ${ }^{* * * *}$
}

\begin{abstract}
RESUMO
Objetivo: Analisar a vivência da família no cuidado domiciliar às crianças com necessidades especiais de saúde (CRIANES). Material e método: Trata-se de um estudo qualitativo, onde foram analisados 134 prontuários em uma instituição filantrópica em um município de Minas Gerais e realizadas dez entrevistas semi-estruturadas com famílias de CRIANES. A análise dos dados foi realizada por meio da análise de conteúdo proposta por Bardin. Resultados: Os resultados sinalizam a complexidade presente nos cuidados realizados com a CRIANES e foi possível identificar uma sobrecarga do cuidador principal. Conclusão: Evidencia-se a importância da efetivação de políticas públicas para esse grupo infantil e a necessidade de uma consolidação da rede social e de apoio para favorecer o acesso e utilização oportuna aos serviços e para melhorias na qualidade de vida das CRIANES e suas famílias
\end{abstract}

Palavras chave: Doença crônica, saúde da criança, cuidadores, enfermagem familiar.

\footnotetext{
ABSTRACT

Objective: The objective of the research is to analyze the family's experience with home care for children with special health needs (CRIANES). Method: This is a qualitative study, which analyzed 134 records at a philanthropic institution in a district of Minas Gerais and ten semi-structured interviews were performed to families of children with special health needs. Data analysis was performed using the content analysis proposed by Bardin. Results: The results indicate the complexity underlying the care provided to children with special

* Graduada em Enfermagem pela Universidade Federal de São João Del Rei. Divinópolis, Brasil. Email: kamillamilione@ yahoo.com.br

${ }_{* \star}$ Graduada em Enfermagem pela Universidade Federal de São João Del Rei. Divinópolis, Brasil. Email: gabi-vilaca@hotmail.com.

${ }^{* * *}$ Graduada em Enfermagem pela Universidade Federal de São João Del Rei. Divinópolis, Brasil. Email: thais_ptu1@hotmail.com.

${ }_{* * * *}$ Graduada em Enfermagem pela Universidade Federal de São João Del Rei. Divinópolis, Brasil. Email: gabrielalomba2@ hotmail.com.thais_ptu1@hotmail.com.

${ }^{* * * * *}$ Doutora em Enfermagem. Professora Adjunta da Universidade Federal de São João Del Rei. Divinópolis, MG, Brasil. Email: patriciabragaenf@ig.com.br
} 
health needs and it was also possible to identify an overload that falls on the primary caregiver. Conclusion: The importance of effective implementation of public policies for this group of children is evident and the need for the strengthening of the social and support networks to facilitate access and timely use of the services in order to improve the quality of life of the children with special health needs and their families.

Key words: Chronic disease, child health, caregivers, family nursing.

\section{RESUMEN}

Objetivo: Analizar la vivencia de la familia en el cuidado domiciliar a los niños con necesidades especiales de salud. Método: Se trata de un estudio cualitativo, donde se analizaron 134 registros en una institución filantrópica en un municipio de Minas Gerais y se realizaron diez entrevistas con familias de niños con necesidades especiales de salud. El análisis de los datos fue realizado por medio del análisis de contenido propuesto por Bardin. Resultados: Los resultados señalan la complejidad presente en los cuidados realizados con los niños con necesidades especiales de salud y fue posible identificar una sobrecarga del cuidador principal. Conclusión: Se evidencia la importancia de la aplicación efectiva de políticas públicas para ese grupo de niños y la necesidad de una consolidación de la red social y de apoyo para favorecer el acceso y utilización oportuna de los servicios y para mejoras en la calidad de vida de los niños y sus familias.

Palabras claves: Enfermedad crónica, salud del niño, cuidadores, enfermería familiar.

Fecha recepción: 18/05/15 Fecha aceptación: 21/01/17

\section{INTRODUÇÃO}

Nos últimos anos, houve uma redução na taxa de mortalidade infantil por doenças infecciosas e imunopreviníveis, devido aos avanços tecnológicos e a implementação de estratégias e ações de saúde direcionadas a esse quadro de morbimortalidade. Em contrapartida, persistem altas taxas de adoecimento e morte por afecções perinatais (1).

As crianças sobreviventes frente às afecções perinatais são caracterizadas por apresentarem repercussões e consequências durante o curso da vida e adquirirem algum tipo de necessidade diferenciada em relação a outras crianças, que são conhecidas como condições crônicas de saúde. Tais crianças, sobreviventes aos avanços tecnológicos na área da saúde, mudaram o perfil epidemiológico da infância, com o aumento das taxas de morbimortalidade devido as condições crônicas na infância (2).

De acordo com Stein et al. (3), a condição crônica na infância é de base biológica, psicológica ou cognitiva de longa duração que produzem consequências como: 1 . limitações de função, atividade ou papel social, se comparadas com crianças saudáveis da mesma idade; 2. dependência de mecanismos compensatórios e cuidados que possibilitem minimizar as limitações de função, atividades ou de desempenho do seu papel social e 3 . uso de serviços acima do usual para a saúde e idade da criança, como médicos, psicológicos, educacionais, e/ou tratamentos especiais em curso e acomodações modificadas em casa ou na escola.

Esse perfil de morbidade contribuiu para o surgimento de uma clientela inédita para os serviços de saúde e representa um novo desafio para as famílias. Essas crianças são consideradas como clinicamente frágeis e socialmente vulneráveis, pois estão sujeitas a internações prolongadas, inúmeras reinternações e aumento da complexidade diagnóstica (4).
A Maternal and Health Children Bureau, 
nos Estados Unidos, define esse grupo de crianças como Children with Special Health Care Needs (CSHCN). Essa terminologia foi adotada na literatura internacional para designar as crianças com estado de saúde frágil e dependente de cuidados de saúde contínuos para assegurar sua sobrevivência (5). No Brasil, elas foram denominadas de Crianças com Necessidades Especiais de Saúde (CRIANES), sendo conhecidas também como herdeiras da tecnologia (6).

As CRIANES caracterizam-se por apresentarem condições especiais de saúde com demandas de cuidados contínuos, de natureza temporária ou permanente, necessitando de uma assistência em saúde e social para além dos requeridos por outras crianças em geral (7). No Brasil, elas foram classificadas de acordo com a tipologia de cuidados requeridos, em cinco grupos. No primeiro, estão incluídas as crianças com disfunção neuromuscular que requerem reabilitação psicomotora e social. No segundo, aquelas dependentes de tecnologia. No terceiro, estão as farmacodependentes. No quarto, as que dependem de modificações na forma habitual de se cuidar, incluindo a realização de tarefas comuns do dia a dia. No último, as que apresentam duas ou mais dessas demandas juntas $(8,9)$.

A condição crônica na infância é um assunto relevante para a reflexão do processo de enfrentamento da família diante das repercussões e consequências que essa condição impõe na vida da criança e de sua família (6). Portanto, o estudo justifica-se em razão da complexidade e continuidade do cuidado presente no cotidiano das CRIANES e suas famílias. Isso é evidenciado pelas dificuldades e sobrecarga familiar no cuidado domiciliar à estas crianças (6), o que mostra a pertinência de desenvolver uma pesquisa que pode revelar os desafios e as potencialidades do cuidado à CRIANES no município cenário. Diante disso, o objetivo da pesquisa é analisar a vivência da família no cuidado domiciliar às CRIANES.

\section{MATERIAL E MÉTODO}

Trata-se de um estudo qualitativo, no qual permite ter uma compreensão subjetiva e particular dos sujeitos, com base em uma experiência concreta, vivenciada no cotidiano da vida, oferecendo detalhes dos contextos locais e momentos no tempo singular (10).

A pesquisa foi realizada no período de novembro de 2013 a julho de 2014 e foi dividida em duas etapas. A primeira etapa foi realizada em uma instituição filantrópica de um município da região Centro Oeste de Minas Gerais, que oferece atendimento pedagógico nas modalidades de educação infantil, educação estruturada, oficinas terapêuticas e também oferece atendimentos ambulatoriais por uma equipe multiprofissional para pessoas com deficiência intelectual e múltipla.

Inicialmente foi realizado o mapeamento de todas as crianças atendidas pela instituição, utilizando-se um questionário elaborado pelas pesquisadoras, que permitiu identificar o perfil de morbidade das crianças assistidas. Dessa forma, foram utilizados como fonte de informação 134 prontuários e coletadas informações como: naturalidade, sexo da criança, diagnóstico/patologia, tipo de parto, intercorrências gestacionais, complicações neonatais e pós-neonatais, escala de apgar, perfil econômico, tipologia de cuidados requeridos pela criança e dependência nas atividades de vida diária.

A análise descritiva dos dados levantados nesta etapa foi realizada após a tabulação em banco de dados utilizando o Programa SPSS - Statistical Package for the Social Sciences que, além de traçar o perfil dos usuários do serviço, também identificou aquelas que atenderiam aos critérios de inclusão.

Os critérios de inclusão definidos para a segunda etapa foram crianças dependentes nas atividades da vida diária além do esperado pela idade (higiene, alimentação e locomoção), que fazem parte do quinto grupo de classificação de tipologia de cuidados reque- 
ridos (dois ou mais tipos de dependências) proposto por Rezende e Cabral (8) e Fereday et al. (9), por permitir revelar a complexidade do cuidado domiciliar inerente a este perfil de CRIANES e as que os membros da família exerçam um cuidado direto a elas com idade superior a 18 anos e capacidade civil plena.

Dessa forma, foram identificadas trinta e sete CRIANES que atendiam aos critérios de inclusão. Em seguida, as pesquisadoras entraram em contato com as famílias para o convite de participação no estudo. Nesta fase, as famílias que aceitaram o convite participaram de uma entrevista semiestruturada, após a assinatura do Termo de Consentimento Livre e Esclarecido.

A entrevista semi-estruturada apresenta relativa flexibilidade, pois apresentam questionamentos pré-formulados pelos autores, porém permite que o entrevistado tenha liberdade de abordar o tema proposto sem se prender à pergunta estabelecida (11).

Foram realizadas no total dez entrevistas com as famílias de CRIANES. A interrupção das entrevistas se deu mediante a saturação de informações dos diferentes núcleos familiares. A saturação amostral tem como objetivo finalizar a coleta de dados ou definir a quantidade de participantes que irão participar da pesquisa $(12,13)$.

A análise dos dados qualitativos se deu por meio da análise de conteúdo temático proposta por Bardin (14). Dessa forma, as entrevistas foram transcritas na íntegra e, em seguida codificadas para que fosse garantido o anonimato dos participantes. Realizou-se primeiramente uma leitura flutuante dos discursos e depois uma leitura exaustiva, a fim de levantar as principais informações evidenciadas pelas famílias. Em seguida, os trechos dos discursos foram agrupados conforme a semelhança de temas permitindo a construção de duas categorias: Os cuidados especiais dispensados à criança e as reorganizações familiares; a atenção à saúde e cuidados profissionais requeridos pelas crianças com necessidades especiais. Através delas foi realizada a interpretação dos dados com embasamento no referencial teórico, permitindo a construção de um texto de análise.

A pesquisa foi desenvolvida respeitando a Resolução 466/12 do Conselho Nacional de Saúde, garantindo as exigências previstas, como sigilo, anonimato e o uso do material exclusivo para fins científicos. O estudo foi aprovado pelo Comitê de Ética em Pesquisa envolvendo seres humanos da Universidade Federal de São João Del Rei, Campus Centro Oeste, obtendo protocolo de número 21819313.2.0000.5545.

\section{RESULTADOS}

A análise dos 134 prontuários, permitiu descrever o perfil de morbidade do grupo infantil atendido pela instituição e identificar crianças com alto grau de dependência para as atividades de vida diária que foram incluídas na etapa das entrevistas semi-estruturadas. A partir dos dados, revelou-se que $84,3 \%$ das crianças assistidas eram naturais do município cenário. Em relação ao sexo, $56,7 \%$ das crianças eram do sexo masculino. Dentre o perfil de morbidade deste grupo infantil, $26,9 \%$ das crianças apresentavam Paralisia Cerebral Espástica, 14,2\% tinham Atraso no Desenvolvimento Neuropsicomotor (ADNPM), 12,7\% Síndrome de Down, 10,4\% Autismo, as demais crianças apresentavam outras morbidades. Ressalta-se que muitas delas apresentavam mais de um tipo de morbidade, revelando a complexidade presente no cotidiano dessa clientela.

A análise do conteúdo das entrevistas permite apresentar a seguir duas categorias empíricas: Os cuidados especiais dispensados à criança e as reorganizações familiares; a atenção à saúde e cuidados profissionais requeridos pelas crianças com necessidades especiais.

1. Os cuidados especiais dispensados à 
criança e as reorganizações familiares.

A análise dos discursos revelou que as famílias passaram por momentos difíceis e sentimentos diversos no momento da descoberta da condição da criança, onde os sentimentos como negação, dúvida, medo, desespero, preocupação, mágoa, tristeza e indignação estavam presentes. A análise indica que o diagnóstico da necessidade especial de saúde da criança pelas famílias ocorreu, na maioria dos casos, após o nascimento da criança, sendo que algumas conseguiram obtê-lo imediatamente e outras só tomaram conhecimento da condição crônica após alguns meses. Conforme pode ser identificado nos enunciados a seguir:

"No começo, eu tive muitas dúvidas, mas assim, pra poder orientar foi difícil achar uma pessoa pelo fato dele ter uma doença rara (A)."

"Eu sempre ficava triste, mas nunca desanimava. Corria lá ele estava bem, chegava ao outro dia ele tinha dado uma recaída, aí ficava abalada $(J)$ ”.

Em virtude da necessidade especial de saúde, essas crianças necessitam de cuidados integrais além do esperado para a idade, que faz com que elas se tornem totalmente dependentes de um cuidador para as atividades de vida diária. Observou-se que elas não conseguem se alimentar sozinhas; não conseguem se locomover; dependem de alguém para realizar os cuidados de higiene; são dependentes de medicamentos; equipamentos, como cadeiras de rodas, órteses, sonda gástrica, parapodium e aspirador; e além disso, necessitam de uma terapêutica de reabilitação constante. $\mathrm{O}$ que pode ser evidenciado nos seguintes enunciados:

"Ela tem uma sonda gástrica, mas ela usou muito tempo a nasoentérica (I).”

O cuidador principal, que durante as entrevistas foi evidente ser a mãe, altera sua rotina para cuidar da criança e na maioria das vezes é obrigada a abandonar o trabalho e outras atividades da sua vida diária para se dedicar exclusivamente aos cuidados à criança. Isto permite inferir que há uma sobrecarga gerada em torno desta mãe frente à complexidade de tarefas que é demandada a ela. Durante os relatos, percebe-se que além dos cuidados com a criança com necessidade especial, o cuidador principal é responsável por cuidar da casa e dos outros filhos. Esta análise sinaliza que este contexto exaustivo gera prejuízos para a sua qualidade de vida:

"Eu tenho que ficar o tempo todo com ela, então não dá pra trabalhar, não dá pra estudar, não dá pra fazer nada nesse sentido. É uma criança especial, então exige muito cuidado, acho que não tem um lado fácil, porque você tem que tá ali 24 horas e ela é totalmente dependente de mim, pra tudo (I)".

Apesar do relato de sobrecarga nos cuidados prestados às crianças com condições crônicas, existe uma predominância de famílias que relatam não haver diferença na maneira de se relacionar entre as CRIANES e os irmãos, ou outras crianças em geral, porém, uma família contradiz essa afirmativa:

\section{"Eu não vejo diferença do meu filho para outras crianças não. E o cuidado assim, é só um pouco mais lento $(A)$ ". \\ "Tem, a diferença tem, né?! Nossa, é muita dife- rença, em tudo (B)”.}

Outro aspecto evidenciado na análise diz respeito à renda familiar, que também sofre alterações devido ao abandono do emprego de um membro da família e devido ao aumento de gastos com a criança que requer atendimentos em saúde e medicamentos contínuos para a sua reabilitação. Entretanto, em alguns momentos, as famílias conseguem os equipamentos necessários ao recorrerem à judicialização para sua obtenção. Isto se mostra evidente nos seguintes discursos: 
"Compramos tudo que precisa pra ele, tutor, cadeira, colar, medicamento (B)".

"Pela prefeitura e pelo Estado a gente teve que entrar no judiciário pra poder conseguir (D)”.

A análise das entrevistas sinaliza que há necessidade de alterações na estrutura física do domicílio para facilitar a acessibilidade da criança. Algumas famílias percebem a importância da reestruturação da casa, contudo, a falta de recursos financeiros dificulta a realização dessas mudanças em alguns casos. Em contrapartida, em uma das entrevistas identificamos que não adaptar o domicílio é compreendido, pela cuidadora, como uma forma de promover a independência e a adaptação da criança no domicílio diante da sua necessidade especial de saúde, visto que ele poderá sentir dificuldade de acessibilidade ao se deslocar do seu domicílio:

"Eu e o pai dele, a gente pensa da seguinte forma: se a gente adaptar tanto a nossa casa, o mundo não vai adaptar por causa dele, ele que tem que adaptar (...) ele tem a visão bem bai$x a$, então a gente pretende colocar rampa onde tem escada, essas coisas a gente pretende facilitar pra ele, mas assim nem tanto sabe. A gente não pode mudar tanta coisa porque ele precisar criar uma independência, porque não adianta ele ter tudo fácil aqui dentro e na hora que ele sair ele não conseguir, então a gente não modificou tanta coisa não (A)”.

Apesar de todas as dificuldades vivenciadas pelas famílias no cuidado às CRIANES, identifica-se nos discursos que com o passar do tempo há uma adaptação da família em relação aos cuidados rotineiros e o compartilhamento dos conhecimentos adquiridos nessa vivência com outras pessoas:

"Eu estou chegando à conclusão que com eu lidar com ele no dia a dia, eu que estou ensinando, até para as pessoas que estão trabalhando com ele na escola $(G)$ ".
A análise revelou que as famílias buscam através da espiritualidade, força e esperança para amenizar os desafios vivenciados, crendo que a fé ajudará na evolução do prognóstico da criança. Também revela que a experiência de conviver com uma criança com necessidade especial de saúde contribui de forma positiva para o crescimento pessoal dos cuidadores:

"Eu tenho muita esperança, muita fé em Deus, que eu vou ver ele ainda fazendo muitas coisas que hoje mesmo ele já me mostra a diferença $(J)^{\prime \prime}$.

2. A atenção à saúde e cuidados profissionais requeridos pelas crianças com necessidades especiais

A análise revela que o cotidiano de cuidados com a criança é complexa, visto que além dos cuidados integrais realizados no domicílio, a criança necessita de cuidados realizados por uma equipe multiprofissional, que é desenvolvido com atendimentos realizados pelos profissionais do serviço cenário deste estudo. Essa instituição conta com uma equipe multidisciplinar para favorecer o desenvolvimento dessas crianças e orientar as famílias. Foi possível perceber através da análise dos discursos, que além do apoio recebido por este serviço, três famílias contam com atendimentos particulares de fisioterapia, recebendo visitas domiciliares e assistência domiciliar quando necessário. Apenas uma família relata contar com o apoio da atenção primária em saúde (APS), as outras famílias relatam que não recebem um atendimento resolutivo na APS, por isso não o procuram mais. Isso revela uma fragilidade na atenção a estas crianças pela APS, e uma dificuldade de acesso ao SUS, visto que apenas uma família relatou a participação deste serviço no cuidado a CRIANES:

"A rotina é bem corrida né, tem que cumprir horário na instituição, eu saio com ele todos os 
dias para a terapia né, todos os dias, de segunda à sexta $(H)$ ".

"Não precisa ir mais ao posto de saúde, eles vem aqui, tá até do lado de casa, pertinho, eles vem direto quando precisa, também é só comunicar lá e eles vem dar assistência (J)”.

A análise das entrevistas evidenciou que todas as cuidadores realizam estimulações do desenvolvimento neuropsicomotor da criança no domicílio e a maioria das famílias reconhece a importância da estimulação na vida dessas crianças, demonstrando através de comparações, o que a criança não fazia antes e o que começou a fazer após as estimulações. Porém, as famílias encontram dificuldades na realização dos procedimentos, na adaptação dos cuidados no domicílio e na compreensão das orientações, visto que as CRIANES demandam cuidados que fazem parte do campo do saber e prática dos profissionais da saúde, este achado pode ser identificado nos enunciados a seguir:

"De alguns meses atrás até agora, ele já me dá um sorriso, ele entende, você conversa perto dele, ele te ouve, ele ri, coisa que ele não fazia $(J)$ ".

"Pra mim é uma coisa muito dolorosa, uma mãe pegar e colocar uma coisa ali dentro do nariz, sabendo que é desagradável, só que sabendo que é pro bem dele mesmo $(F)$.”

\section{DISCUSSÃO E CONCLUSÃO}

A análise dos dados permite inferir que a descoberta da condição crônica de saúde na infância pela família é uma situação que provoca mudanças profundas na convivência e causa alterações nas relações intrafamiliares, marcadas por sentimentos de medo, insegurança e comportamentos diversos, o que também foi verificado em outros estudos $(15,16)$. A família passa por períodos de adaptação, em razão da demanda de cuida- dos especiais que estas crianças necessitam e desencadeia uma desorganização inicial intensa em sua rotina (16). Porém, com o passar do tempo, os familiares adquirem experiência de como lidar com as limitações da criança no seu dia a dia, o que contribui para a reorganização das tarefas desenvolvidas no cuidado.

Os resultados permitiram visualizar a dificuldade presente nos cuidados realizados com a CRIANES, pelo fato de muitas vezes exigirem habilidades que são adquiridas com a prática vivenciada. Os cuidadores enfrentam diferentes demandas de tarefas, que envolvem até mesmo cuidados de enfermagem, os quais a família precisa incorporar ao seu cotidiano de cuidar, para além daqueles pertinentes às crianças em geral. Nishimoto e Duarte (17) revelam em seu estudo que o enfermeiro envolvido no cuidado a essas CRIANES deve traçar estratégias para facilitar o cuidado desenvolvido no domicílio pelas famílias, promovendo uma assistência de qualidade em prol dessas crianças. O estudo realizado por Neves et al. (18) evidencia os cuidados e habilidades que são desenvolvidos no cotidiano das CRIANES, como por exemplo, na alimentação de uma criança que possui sonda gástrica e na locomoção de uma criança usuária de cadeira de rodas, onde há necessidade dos familiares cuidadores superarem os desafios da acessibilidade e mobilidade. Portanto, segundo Elias e Murphy, otimizar a saúde da criança e minimizar as internações recorrentes ou prolongadas é o principal objetivo dos cuidados de saúde em casa (19).

A necessidade especial de saúde da criança gera, portanto, um acúmulo de tarefas sobre os principais cuidadores, que na maioria das vezes é a mãe, seguida por outras mulheres da família $(18,20)$. A análise dos dados permitiu compreender que o cuidador principal, muitas vezes, fica sobrecarregado devido à dedicação exclusiva para com a CRIANES, além de ser responsável pelas funções domésticas e o cuidado com os outros filhos, o que limita 
suas atividades sociais e interfere na atenção voltada para com os outros membros da família. Castro e Piccinini (21) afirmam que ao acompanhar o filho doente, a mãe se distancia dos outros membros da família, tornando uma experiência desestruturante para todos os membros e um momento de rompimento dos laços familiares. Dessa forma, é importante oferecer oportunidades de atualizar e ampliar as habilidades necessárias para o cuidado domiciliar das CRIANES para outros membros da família, como os avós (19).

Além disso, o abandono do emprego pelo cuidador principal tornou-se evidente durante os depoimentos, considerando a dependência total da criança no cotidiano. $\mathrm{O}$ abandono do vínculo empregatício desencadeia uma série de frustações e preocupações aos membros da família, pois acaba gerando alterações na renda familiar. Um estudo realizado por Looman et al. demonstra que as condições de saúde das CRIANES aumentam as despesas médicas aproximadamente duas a três vezes mais do que quando comparadas de crianças sem limitações funcionais. Com isso, as demandas são significativamente maiores sobre o tempo e as finanças de suas famílias (22). Estudos mostram que muitas vezes as mães assumem a responsabilidade pela adequação das suas atividades com as necessidades de seus filhos, cabendo à elas a desistência do emprego para melhor atender às demandas da criança doente. Essa modificação na dinâmica familiar provoca redução do orçamento, podendo elevar os níveis de estresse no relacionamento da família (23).

Sabe-se que as crianças com necessidades especiais de saúde necessitam de uma reabilitação terapêutica dinâmica, essencial para o seu desenvolvimento neuropsicomotor. Os discursos permitiram compreender que as crianças participantes do estudo recebem acompanhamento terapêutico de uma instituição voltada pra este tipo de clientela, que é a instituição coparticipante desta pesquisa. Esse apoio recebido pelas famílias contribui para a reabilitação da criança e é fonte de orientação das famílias no que se diz respeito às necessidades especiais da criança. As famílias contam ainda com atendimentos domiciliares e/ou outros serviços particulares, que complementam a terapêutica e contribuem para um melhor desenvolvimento da criança.

Estudos mostram que a condição crônica exige tratamento de uma equipe multidisciplinar ao longo da vida, que precisa ser iniciado de forma precoce e contínua, requerendo serviços especializados que garantam assistência qualificada. Porém, Figueiredo et al. apresentam que a equipe de saúde se mostra despreparada para lidar com a família de crianças com necessidades especiais (24). O estudo de Tavares (25) revelou que devido a deficiência na oferta e gestão dos serviços de saúde acontece a interrupção ou o atraso do acompanhamento das crianças. A Política Nacional de Saúde da pessoa portadora de deficiência estabelece como competência do Sistema Único de Saúde (SUS) viabilizar a integralidade da atenção aos portadores de deficiência, propiciando acesso às ações de saúde específicas da condição, inclusive concessão de órteses e próteses, além das ações voltadas às doenças e agravos comuns à infância (26).

Os discursos apontaram que apenas uma família recebe assistência da atenção primária de saúde, o que permite pressupor que existem fragilidades na rede de atenção no contexto do SUS e nas políticas públicas voltadas para esse grupo infantil. Uma pesquisa realizada no Brasil, revela que as famílias apresentam dificuldades de acesso aos serviços de saúde (27). Outro estudo realizado no sul do Brasil apontou que os familiares cuidadores ao buscarem os serviços de atendimento primário de saúde, se deparam com um sistema que não é resolutivo, e frequentemente esses familiares são encaminhados para o domicílio sem resposta às suas necessidades (28). Nóbrega afirma que, o relacionamento entre a equipe que atua na Estratégia de Saúde da família (ESF) e as mães não é sempre caracterizado por acolhimento, acessibilidade e 
satisfação e as famílias demonstraram insatisfação com o atendimento dos profissionais de saúde da ESF, repercutindo negativamente na formação de vínculo e na atenção à criança com necessidade especial de saúde (29).

Pode-se perceber que grande parte das famílias busca apoio para o enfrentamento dos desafios vivenciados no âmbito espiritual. Estudos mostram que a crença em algo maior é importante uma vez que possibilita aos indivíduos o enfrentamento de situações adversas e a superação de sentimentos negativos gerados no curso da doença. Dessa forma, a espiritualidade tem contribuído para a melhora do estado emocional por trazer esperança, perdão, altruísmo e amor (16). O estudo permite inferir que as famílias encontram significados nas crenças religiosas para amenizarem o sofrimento e enfrentarem a condição crônica do filho com serenidade e segurança.

Pode-se concluir que com a descoberta da necessidade especial da criança, os cuidadores passam a ter uma vida social restrita, em decorrência da necessidade de atenção integral e cuidados complexos exigidos pelas CRIANES, gerando assim mudanças na rotina do núcleo familiar. O processo de adaptação vivenciado pela família é permeado, na maioria das vezes por sentimentos negativos, que são amenizados pela busca do apoio na espiritualidade para superação dos desafios enfrentados.

Muitos dos cuidadores abandonam o emprego e consequentemente tem um déficit na renda familiar, que se reforça devido à aquisição de medicamentos, materiais e equipamentos de saúde que nem sempre são adquiridos pelo Sistema Único de Saúde. Outra evidência foi a fragilidade no atendimento domiciliar pela atenção primária em saúde, que se faz importante fonte de apoio para as famílias no cuidado domiciliar às CRIANES. Dessa forma percebe-se a necessidade do acolhimento humanizado, resolutividade e a criação de vínculos entre a família e a equipe de saúde para que se tenha uma assistência adequada a essas crianças, favorecendo atenção oportuna às mesmas.

Assim, pode-se concluir que o enfermeiro deve desempenhar um importante papel frente às ações direcionadas às CRIANES, visto que ele atua como articulador de toda a equipe de saúde, estando devidamente preparado e capacitado para atuar de maneira resolutiva nas dificuldades e problemas enfrentados pelas famílias e CRIANES.

Nesse sentido, evidencia-se a importância da efetivação de políticas públicas e a evolução de práticas de saúde, como a implementação de um modelo de atenção direcionado às CRIANES, educação permanente para os profissionais de saúde e uma formação profissional adequada para atender de maneira resolutiva as demandas e necessidades de saúde/doença desse grupo infantil. Além da necessidade de uma consolidação de uma rede de apoio efetiva, a fim de minimizar a sobrecarga da família.

\section{REFERÊNCIAS}

1. Departamento de Informática do Sistema Único de Saúde (DATASUS) [Internet]. Brasília (DF): Ministério da Saúde, Brasil/SUS; 2006 [citado 15 out 2016]. Disponível em: http://www. datasus.gov. br.

2. Neves ET, Silveira A, Arrué AM, Pieszak GM, Zamberlan KC, Santos RP. Rede de cuidados de crianças com necessidades especiais de saúde. Texto contexto-enferm. 2015; 24(2): 399-406.

3. Stein RE, Bauman LJ, Westbrook LE, Coupey SM, Ireys HT. Framework for identifying children who have chronic conditions: the case for a new definition. J Pediatr. 1993; 122(3): 342-347.

4. Neves ET, Cabral IE. A fragilidade clínica e a vulnerabilidade social das crianças com necessidades especiais de saúde. Rev Gaucha Enferm. 2008; 29(2): 182-90.

5. McPherson M, Arango P, Fox H, Lau- 
ver $C$, McManus $M$, Newacheck $P$, et al. A new definition of children with special health care needs. Pediatrics. 1998; 102(1): 137-41.

6. Cabral IE, Vernier ETN. Caracterização de crianças com necessidades especiais de saúde e seus familiares cuidadores. Santa Maria (RS). 2004-2005: Subsídios para intervenções de enfermagem. Rev. Soc. Bras. Enferm. Ped. 2006; 6(1): 37-45.

7. Cabral IE, Silva JJ, Zillmann DO, Moraes JR, Rodrigues EC. A criança egressa da terapia intensiva na luta pela sobrevida. Rev Bras Enferm. 2004; 57(1): 35-9.

8. Rezende JMM, Cabral IE. As condições de Vida das Crianças com Necessidades Especiais de Saúde: determinates da vulnerabilidade social, na Rede de Cuidados em Saúde as crianças com Necessidades Especiais de Saúde. R. pesq.: cuid. fundam. online [Internet]. 2010 [citada 1 mar 2015]; 2 (Ed. Supl.): 22-5. Disponível em: http://www.seer.unirio.br/index.php/cuidadofundamental/article/ view/773/pdf_68

9. Fereday J, Oster C, Darbyshire P. Partnership in practice: what parents of a disabled child want from a generic health professional in Australia. Health Soc Care Community. 2010; 18(6): 624-32.

10. Pope C, Mays N, organizadores. $3^{\mathrm{a}}$ ed. Pesquisa qualitativa na atenção à saúde. Porto Alegre: Artmed; 2009. 117 p.

11. Minayo MCS, Deslandes S, Neto O, organizadoras. Pesquisa social: teoria, método e criatividade. Petrópolis: Vozes; 2011. $154 \mathrm{p}$.

12. Fontanella BJB, Ricas J, Turato ER. Amostragem por saturação em pesquisas qualitativas em saúde: contribuições teóricas. Cad Saude Publica. 2008; 24(1): 17-27.

13. Fontanella BJB, Luchesi BM, Saidel MGB, Ricas J, Turato ER, Melo DG. Amostragem em pesquisas qualitativas: proposta de procedimentos para constatar saturação teórica. Cad Saude Publica. 2011; 27(2): 388-394.
14. Bardin L. Análise de conteúdo. São Paulo: Edições 70; 2011.73 p.

15. Vieira SS, Bevilacqua MC, Ferreira NMLA, Dupas G. Descoberta da deficiência auditiva pela família: vendo o futuro idealizado desmoronar. Acta Paul Enferm. 2012; 25(Spec No 2): 82-88.

16. Esteves JS, Silva LF, Conceição DS, Paiva ED. Dúvidas de familiares sobre o cuidado de crianças com necessidades especiais de saúde dependentes de tecnologia. Invest Educ Enferm. 2015; 33(3): 547-555.

17. Nishimoto CLJ, Duarte ED. A Organização familiar para o cuidado à criança em condição crônica, egressa da unidade de terapia intensiva neonatal. Texto contexto - enferm. 2014; 23(2): 318-327.

18. Neves ET, Cabral IE, Silveira A. Rede familial de crianças com necessidades especiais de saúde: implicações para a enfermagem. Rev Lat Am Enfermagem 2013; 21(2): 562-570.

19. Elias ER, Murphy NA, Council on Children with Disabilities. Home care of children and youth with complex health care 24 needs and technology dependencies. Pediatrics. 2012; 129(5): 996 -1005.

20. Barbosa TA, Reis KMN, Lomba GO, Alves GV, Braga PP. Rede de apoio e apoio social às crianças com necessidades especiais de saúde. Rev. RENE. 2016; 17(1): 60-6.

21. Castro EK, Piccinini CA. Implicações da doença orgânica crônica na infância para as relações familiares: algumas questões teóricas. Psicol Reflex Crit. 2002; 15(3): 625-35.

22. Looman WS, Presler E, Erickson MM, Garwick AE, Cady RG, Kelly AM, et al. Care coordination for children with complex special health care needs: the value of the Advanced Practice Nurse's Enhanced Scope of Knowledge and Practice. J Pediatr Health Care. 2013; 27(4): 293-303.

23. Silva MAS, Collet N, Silva KL, Moura FM. Cotidiano da família no enfrentamento da condição crônica na infância. Acta 
Paul Enferm 2010; 23(3): 359-65.

24. Figueiredo S, Sousa ACC, Gomes IL V. Menores com necessidades especiais de saúde e familiares: implicações para a Enfermagem. Rev Bras Enferm. 2016; 69(1): 88-95.

25. Tavares TS. A continuidade do cuidado às crianças com condições crônicas egressas de terapia intensiva neonatal: a perspectiva das famílias [Tesis maestria]. Belo Horizonte: Universidade Federal de Minas Gerais Escola de Enfermagem; 2012. 188 p.

26. Ministério da Saúde Brasil. Secretaria de Assistência à Saúde. Coordenação de Atenção a Grupos Especiais. Política Na- cional de Saúde da Pessoa Portadora de Deficiência, 2008.

27. Ramos LDC, Moraes JRMM, Silva LF, Góes FGB. O cuidado materno no domicilio as crianças com necessidades especiais de saúde. Invest Educ Enferm. 2015; 33(3): 492-499.

28. Silveira A, Neves ET. Crianças com necessidades especiais em saúde: cuidado familiar na preservação da vida. Cien Saude Colet. 2012; 11(1): 074-080.

29. Nóbrega VM, Damasceno SS, Rodrigues PF, Reichert APS, Collet N. Atenção à criança com doença crônica na estratégia saúde da família. Cogitare Enferm. 2013 Jan/Mar; 18(1): 57-63. 